\title{
Expression of Sox2 in mature and immature teratomas of central nervous system
}

\author{
Ji Hoon Phi ${ }^{1,2}$, Sung-Hye Park ${ }^{2,3}$, Sun Ha Paek ${ }^{1,2}$, Seung-Ki Kim ${ }^{1,2}$, Yun Jin Lee ${ }^{1,2}$, \\ Chul-Kee Park ${ }^{1,2}$, Byung-Kyu Cho ${ }^{1,2}$, Do-Hun Lee ${ }^{1,2}$ and Kyu-Chang Wang ${ }^{1,2}$ \\ ${ }^{1}$ Department of Neurosurgery, Seoul National University College of Medicine, Seoul, Korea; ${ }^{2}$ Clinical \\ Research Institute, Seoul National University Hospital, Seoul, Korea and ${ }^{3}$ Department of Pathology, Seoul \\ National University College of Medicine, Seoul, Korea
}

\begin{abstract}
Sox2 is a transcription factor that plays a critical role in the maintenance of the self-renewal capability of neural stem cells. This study was undertaken to investigate the expression pattern of Sox2 in mature and immature teratomas of the central nervous system. Sox 2 immunohistochemistry was performed in $\mathbf{1 4}$ cases of central nervous system teratoma: five mature, five immature teratomas, and four mixed germ cell tumors with a prominent teratoma component. Fetal brain tissue was used as a normal control. Immunofluorescence with double labeling of stem cells and neuroglial cell markers was used for phenotyping of Sox2-positive cells. In all cases of immature teratomas, positive reactivity to Sox2 was observed in primitive neuroepithelial tissues. Sox2 was not expressed in mature tissues, except in some cuboidal or columnar epithelium of endodermal origin. In mature teratomas, Sox2 expression was limited to some endodermal epithelium in two cases, and no Sox2 expression was observed in the other three cases. The majority of Sox2-positive neuroepithelial cells also expressed neural stem cell markers, nestin and vimentin. Sox2 and neuronal and oligodendroglial markers were expressed in a mutually exclusive manner. However, mature astroglial cells coexpressed Sox2 and GFAP. In fetal brain, Sox2 was mainly expressed in ventricular and subventricular zones. Since Sox2 is strongly expressed in the primitive neuroepithelial tissues of central nervous system immature teratomas, it may be a useful biomarker for the diagnosis and quantitative grading of central nervous system immature teratomas. Modern Pathology (2007) 20, 742-748; doi:10.1038/modpathol.3800793; published online 27 April 2007
\end{abstract}

Keywords: Sox2; immature teratoma; mature teratoma; primitive neuroepithelial tubule; fetal brain

Mammalian central nervous system development is a highly organized process consisting of cell proliferation, migration, differentiation, and apoptosis. $^{1-3}$ The sex determining region of Y chromosome-related high mobility group box2 (Sox2) is a key transcription factor in developmental neurobiology, and maintains the self-renewal capability of neural stem cells. ${ }^{4-8}$ In particular, Sox2 enables cellular proliferation, while inhibiting neuronal fate commitment. Sox2 is highly expressed in neural stem cells, neural plates, and throughout the full thickness of early stage neural tubes, and later in the ventricular zone of the developing central nervous system. ${ }^{6,7,9,10}$ The transcriptional targets of Sox 2 have not been clearly defined, although there is

Correspondence: Dr K-C Wang, MD, PhD, Department of Neurosurgery, Seoul National University College of Medicine, 28 Yeongeon-dong, Jongno-gu, Seoul 110-744, Korea.

E-mail: kcwang@snu.ac.kr

Received 09 January 2007; revised 13 March 2007; accepted 15 March 2007; published online 27 April 2007 some evidence that Notch signaling pathway mediates the activities of Sox $2 .^{9}$

Central nervous system teratomas are uncommon neoplasms that arise in the suprasellar and pineal regions, and sometimes in the spinal cord. ${ }^{11-13}$ Moreover, teratoma immaturity, classically defined by the presence of primitive neuroepithelial tubules and/or primitive neuroectodermal tissues, indicates a borderline malignancy state between mature teratomas and frank malignant teratomas. ${ }^{11-13}$ In the present study, we aimed to investigate the expression patterns of Sox2 in various tissue components, including primitive neuroepithelial tubules, in teratomas. Although the expressions of Sox2 and its homologues have been studied in many species, little information is available for human tissues. Moreover, since teratoma recapitulates human embryogenesis, ${ }^{14}$ albeit in a disorganized fashion, patterns of Sox2 expression in human tissues are of interest. In particular, in the developing nervous system, Sox2 expression is a dynamic phenomenon. ${ }^{6,7,9}$ In the present study, we attempted to determine the phenotype of Sox2-positive cells 
using a double immunofluorescence labeling approach to elucidate Sox2 expression with respect to neuroglial differentiation.

Although neuroepithelial tubules in immature teratomas can mimic early stage human neural tubes, they are not normal in terms of cytogenetic composition or tissue architecture. Therefore, Sox2 expression patterns in immature teratomas need to be compared with those in normal brains. In the present study, we used human fetal brain as a normal control. Sox2 has been reported to be highly expressed in the ventricular zone and subventricular zone of chicks and rodents. ${ }^{6,7,9}$

The diagnosis of immaturity is of crucial clinical importance because immature teratomas of the central nervous system have a poorer prognosis and require more aggressive treatment than mature teratomas. ${ }^{13,15,16}$ Pathological diagnoses usually rely on morphology, although a small number of immunohistochemical markers are used to discriminate neuroepithelial components. Therefore, we reasoned that if Sox2 were consistently expressed in primitive neuroepithelial components, it would be a good candidate marker for immature teratomas.

\section{Materials and methods}

\section{Tissue Samples}

The paraffin-embedded tumor tissues of 14 cases of central nervous system teratoma were available from the Pathology Database at Seoul National University Hospital, for the period 1995-2004 (Table 1): five cases of mature teratomas, five cases of immature teratomas, and four cases of mixed germ cell tumor (two cases with a prominent mature component and two cases with an immature component). Mixed germ cell tumors with a prominent teratoma component were included because of the small number of pure central nervous system teratomas. Paraffinembedded tissues were sectioned, $4 \mu \mathrm{m}$, using a microtome (Leica, Wetzlar, Germany), and trans- ferred to silane-coated slides. As a positive control, the paraffin-embedded tissues of fetal brain, obtained from an autopsied fetus after a therapeutic abortion at gestational week 14, were used: sections were prepared in an identical manner. This study was approved by the Institutional Review Board of Seoul National University Hospital.

\section{Immunohistochemistry}

After incubation in a $60^{\circ} \mathrm{C}$ dry oven for $1 \mathrm{~h}$, paraffinembedded sections were deparaffinized in xylene and rehydrated through graded ethanols. For antigen retrieval, sections were microwaved in $10 \mathrm{mM}$ sodium citrate buffer ( $\mathrm{pH}$ 6.0) for $5 \mathrm{~min}$. After endogenous peroxidase activity had been blocked in PBS containing $1 \%$ Triton ${ }^{\circledR}$ X-100 (Sigma, St Louis, MO, USA) and $1 \% \mathrm{H}_{2} \mathrm{O}_{2}$ for $30 \mathrm{~min}$, PBS containing $1.5 \%$ normal rabbit serum (Vector Laboratories, Burlingame, CA, USA) was applied for $30 \mathrm{~min}$. Sections were then incubated with antiSox2 antibody (1:100, goat IgG; R\&D Systems, Minneapolis, MN, USA) at $4^{\circ} \mathrm{C}$ overnight, this was followed by treatment with biotinylated anti-goat IgG secondary antibody (1:200, rabbit polyclonal; Vector Laboratories) for $1 \mathrm{~h}$. Positive reactions were visualized using avidin-biotin complex kits (Vector Laboratories) with diaminobenzidine (Sigma). Sections were counter-stained with $0.1 \%$ hematoxylin. Axial brain sections of autopsied fetus containing abundant areas of germinal matrix were used as a positive control. A negative control was prepared by omitting primary antibody, while maintaining all other procedures. Only nuclear staining with clear borders was interpreted as true positive: faint cytoplasmic staining, if present, was deemed negative.

\section{Immunofluorescence}

Deparaffinization and antigen retrieval were performed as described above. Nonspecific antibody

Table 1 Study cases

\begin{tabular}{|c|c|c|c|c|}
\hline Case no. & Sex & Age & Location & Histopathological diagnosis \\
\hline 1 & M & 1 month & Temporal & Immature teratoma \\
\hline 2 & $\mathrm{~F}$ & 4 months & Spinal cord & Immature teratoma \\
\hline 3 & M & 5 years & Pineal & Immature teratoma \\
\hline 4 & M & 2 years & Pineal & Immature teratoma \\
\hline 5 & M & 8 years & Pineal & Immature teratoma \\
\hline 6 & M & 8 years & Pineal & Mature teratoma \\
\hline 7 & M & 8 years & Pineal & Mature teratoma \\
\hline 8 & $\mathrm{M}$ & 1 year & Spinal cord & Mature teratoma \\
\hline 9 & $\mathrm{~F}$ & 2 months & Temporal & Mature teratoma \\
\hline 10 & M & 8 years & Pineal & Mature teratoma \\
\hline 11 & M & 23 years & Pineal & MGCT: immature teratoma $(>90 \%)+$ Germ $(<5 \%)+$ YST $(<5 \%)$ \\
\hline 12 & $\mathrm{~F}$ & 11 years & Pineal & MGCT: immature teratoma $(40 \%)+$ Germ $(<10 \%)+$ EC $(30 \%)+C C(<10 \%)$ \\
\hline 13 & $\mathrm{~F}$ & 14 years & Pineal & MGCT: mature teratoma $(65 \%)+$ Germ $(30 \%)+$ EC $(<5 \%)+$ YST $(<5 \%)$ \\
\hline 14 & M & 11 years & Suprasellar & MGCT: mature teratoma $(>95 \%)+\operatorname{Germ}(<5 \%)$ \\
\hline
\end{tabular}

M, male; F, female; MGCT, mixed germ cell tumor; Germ, germinoma; YST, yolk sac tumor; EC, embryonal carcinoma; CC, choriocarcinoma. 
binding was blocked in PBS with 10\% normal donkey serum (Vector Laboratories) and $0.3 \%$ Triton ${ }^{\circledR}$ X-100 (Sigma) for $45 \mathrm{~min}$. Sections were then incubated with anti-Sox2 antibody (1:50, goat IgG; R\&D Systems) diluted in PBS containing 0.1\% bovine serum albumin (Sigma) at $4^{\circ} \mathrm{C}$ overnight. After rinsing, donkey Alex Fluor ${ }^{\circledR}$ 488-conjugated anti-goat IgG secondary antibody (1:200; Invitrogen, Carlsbad, CA, USA) was applied for $1 \mathrm{~h}$ at room temperature. For double labeling, after blocking in PBS containing 10\% normal goat serum and $0.3 \%$ Triton ${ }^{\circledR} \mathrm{X}-100$, sections were incubated with one of the following primary antibodies at $4^{\circ} \mathrm{C}$ overnight: anti-nestin antibody (1:400, mouse IgG; Chemicon, Temecula, CA, USA), anti-vimentin antibody (1:100, mouse IgG; DAKO, Glostrup, Denmark), anti-glial fibrillary acidic protein (GFAP) antibody (1:100, mouse IgG; Santa Cruz Biotechnology, Santa Cruz, CA, USA), anti-microtubule associated protein 2 (MAP2) antibody (1:400, rabbit polyclonal; Chemicon), anti-Tuj1 antibody (1:400, mouse IgG; Covance, Berkeley, CA, USA), or anti-2' $3^{\prime}$-cyclicnucleotide $3^{\prime}$-phosphodiesterase (CNPase) antibody (1:200, mouse IgG; Abcam, Cambridge, UK). Corresponding secondary antibodies were applied for $1 \mathrm{~h}$ at room temperature. The secondary antibodies used were as follows: Cy3-conjugated goat anti-mouse IgG (1:100; Invitrogen), Cy3-conjugated goat anti-rabbit (1:100; Invitrogen). Sections were mounted with antifading solution containing 4'-6-diamidino2-phenylindole (DAPI) (Vectashield ${ }^{\circledR}$, Vector Laboratories). The sections were observed under a confocal microscope (Zeiss, Oberkochen, Germany).

\section{Results}

In the fetal brain sections (positive control), Sox2 expression was found mainly in the ventricular zone and subventricular zone (Figure 1a). The number of Sox2-expressing cells decreased in the intermediate zone, and Sox2 expression was virtually absent in the cortical plate and marginal zone. A uniform dense nuclear staining pattern was observed along ventricular walls from lateral ventricles to the spinal cord central canal. Sox2-positive cells in ventricular zone and subventricular zone coexpressed nestin (Figure 1b) and GFAP (Figure 1c).

Neuroepithelial tubules in immature teratomas are typically multilayered, small-sized, and are disorganized (Figure 2a). However, individual neuroepithelial tubules recapitulate the basic morphology of developing early stage neural tubes, that is, they have a central lumen, ciliated processes abutting the lumen, an elongated cellular morphology, and a stratified pattern (Figure 2b). In all cases of immature teratomas, Sox2 was robustly expressed in primitive neuroepithelial tubules and surrounding tissues (Figure 2c), but not all the cells in neuroepithelial tubules expressed Sox2. Sox2 was also strongly expressed in hypercellular areas that contained round cells and multiple rosettes and resembled primitive neuroectodermal tumors (Figure 2d). Rare Sox2-positive cells were observed in the midst of mature mesenchymal tissues, and these Sox2-positive cells differed in morphology from those in neuroepithelial tubules and primitive neuroectodermal tumor-like areas, because those in the mature mesenchymal tissues had more cytoplasm and elongated processes and resembled astroglial cells (Figure 2e). Double immunofluorescence for Sox2 and GFAP confirmed that they had an astroglial phenotype (Figure 4e and f). In addition, some endoderm-derived cuboidal or columnar epithelium showed weak to moderate reactivity to Sox2 (Figure 2f).

In five cases of mature teratomas, Sox2 was not expressed in mature tissue except in cuboidal or columnal epithelium of endodermal origin in two cases, in which its intensity was weak or moderate, like that observed in immature teratomas. These Sox2-positive epithelial regions resembled bron-
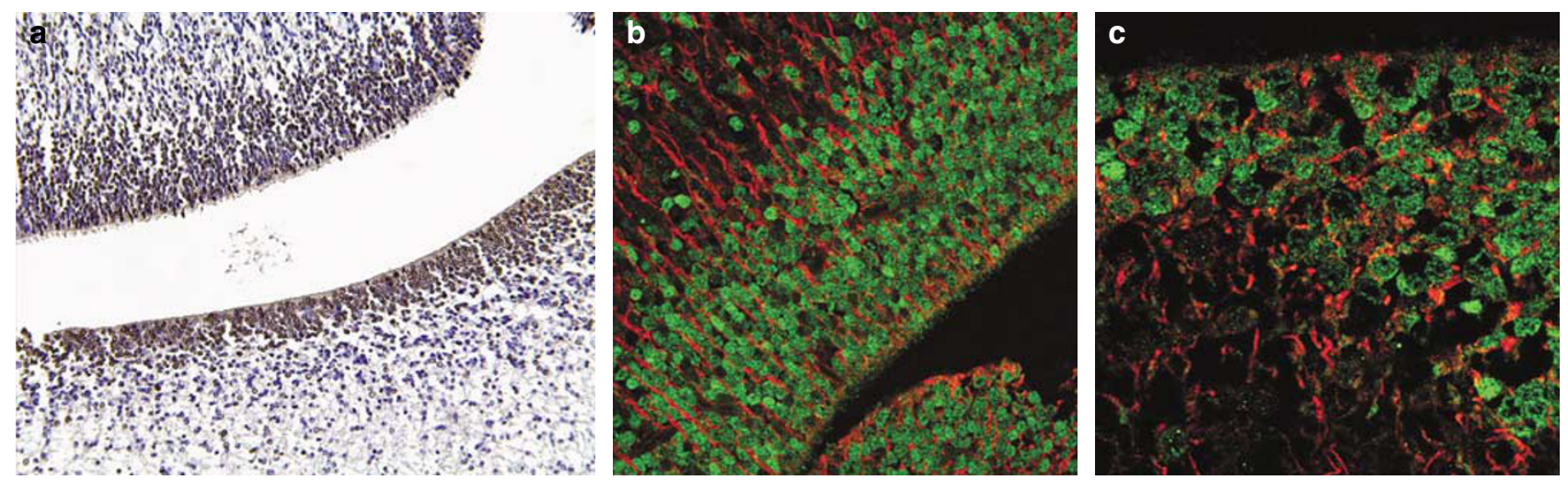

Figure 1 Sox2 expression in fetal brain sections. (a) Sox2 expression in ventricular zone and subventricular zone of the fetal brain. A uniform and dense nuclear staining pattern was observed along ventricular walls (Sox2 immunohistochemistry, $\times 200$ ); $($ b) immunofluorescence studies showed that Sox2-positive cells in ventricular zone and subventricular zone coexpressed nestin (Sox2 green, nestin red, $\times 400$ ); (c) some Sox2-positive cells in ventricular zone and subventricular zone also expressed GFAP (Sox2 green, GFAP red, $\times 800)$. 

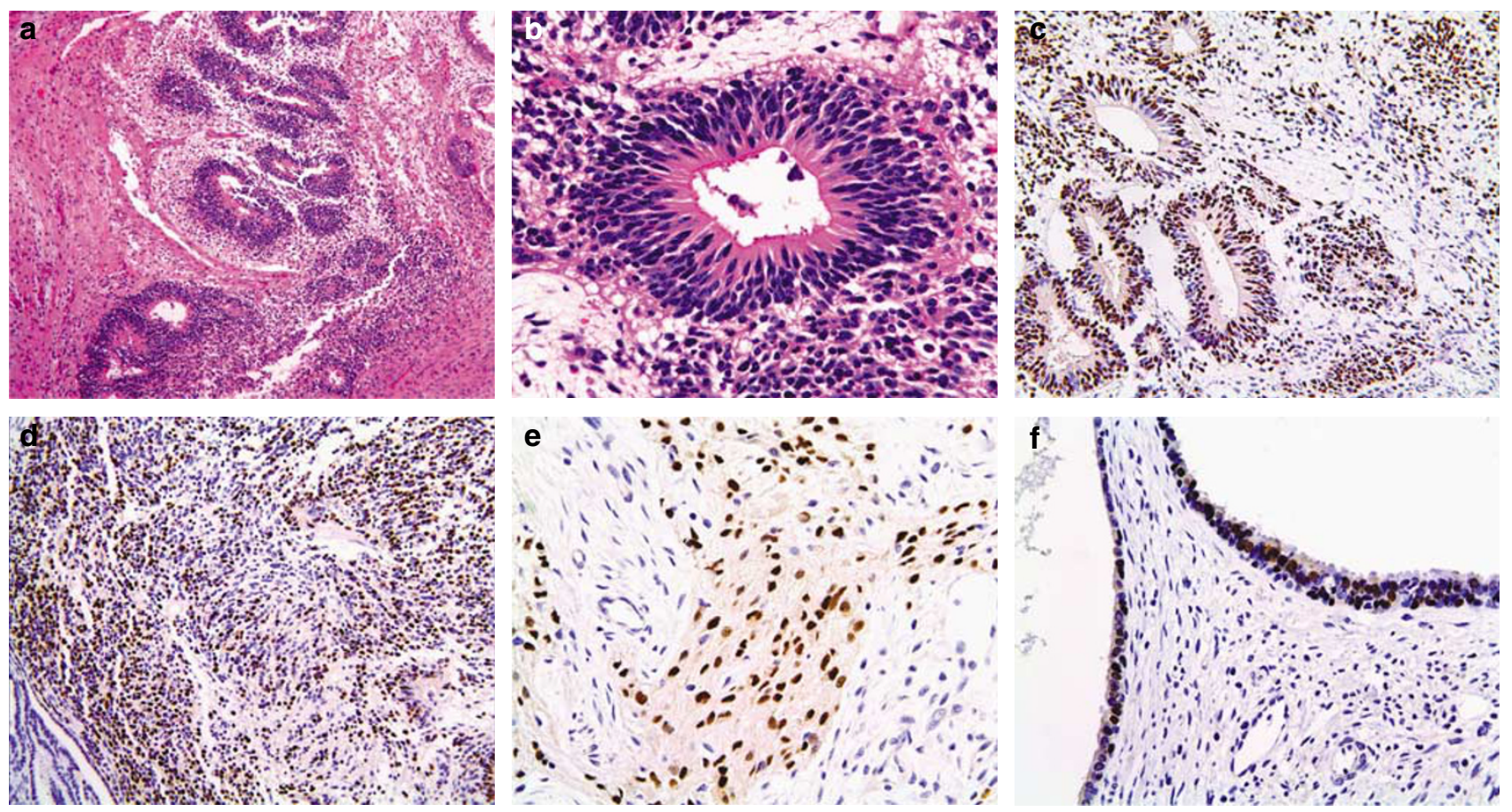

Figure 2 Sox2 expression patterns in central nervous system immature teratomas. (a) The presence of primitive neuroepithelial tubules is a pathognomonic feature of immature teratoma (hematoxylin and eosin, $\times 100$ ); (b) individual neuroepithelial tubules recapitulate the basic morphology of developing early stage neural tubes, and show central lumen, ciliated processes abutting the lumen, an elongated cellular morphology, and a stratified pattern $(\times 400)$; (c) Sox2 was robustly expressed in primitive neuroepithelial tubules and surrounding tissues (Sox2 immunohistochemistry, $\times 200$ ); (d) Sox2 was highly expressed in primitive neuroectodermal tumor-like areas $(\times 200)$; (e) scattered nests of Sox2-positive cells containing much cytoplasm were observed at sites apart from areas containing an abundance of neuroepithelial tubules $(\times 400)$; (f) some cuboidal and ciliated columnar epithelial components of endodermal origin were also Sox2 positive $(\times 400)$
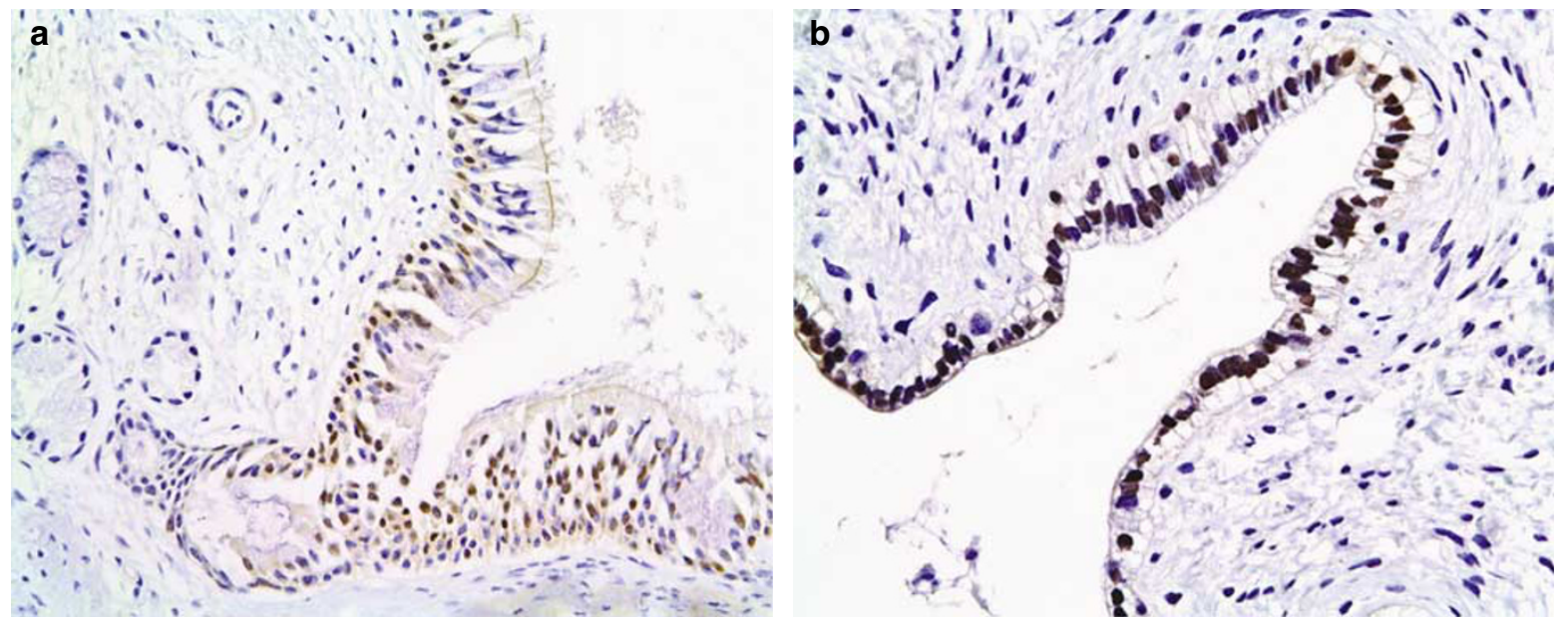

Figure 3 Sox2-positive endodermal epithelium in mature teratomas. (a) Some ciliated columnar epithelium of endodermal origin was Sox2 positive (Sox2 immunohistochemistry, $\times 200$ ); (b) fetal type glandular epithelium of endodermal origin was also found to express Sox2 $(\times 400)$.

chial epithelium (Figure 3a) or fetal type glandular epithelium (Figure 3b). The expression pattern of Sox2 in all cases of mixed germ cell tumor was similar to that described above.

To further characterize Sox2-positive cells, immunofluorescence with double labeling was performed. Sox2-positive cells in the neuroepithelial tubules and cells of the primitive neuroectodermal tumor-like areas coexpressed the neural stem cell markers, nestin and vimentin (Figure 4a-c). However, many Sox2-negative cells also expressed these markers. Sox2-positive cuboidal epithelium did not express nestin, confirming that the cells did not have primitive neuroepithelial characteristics 

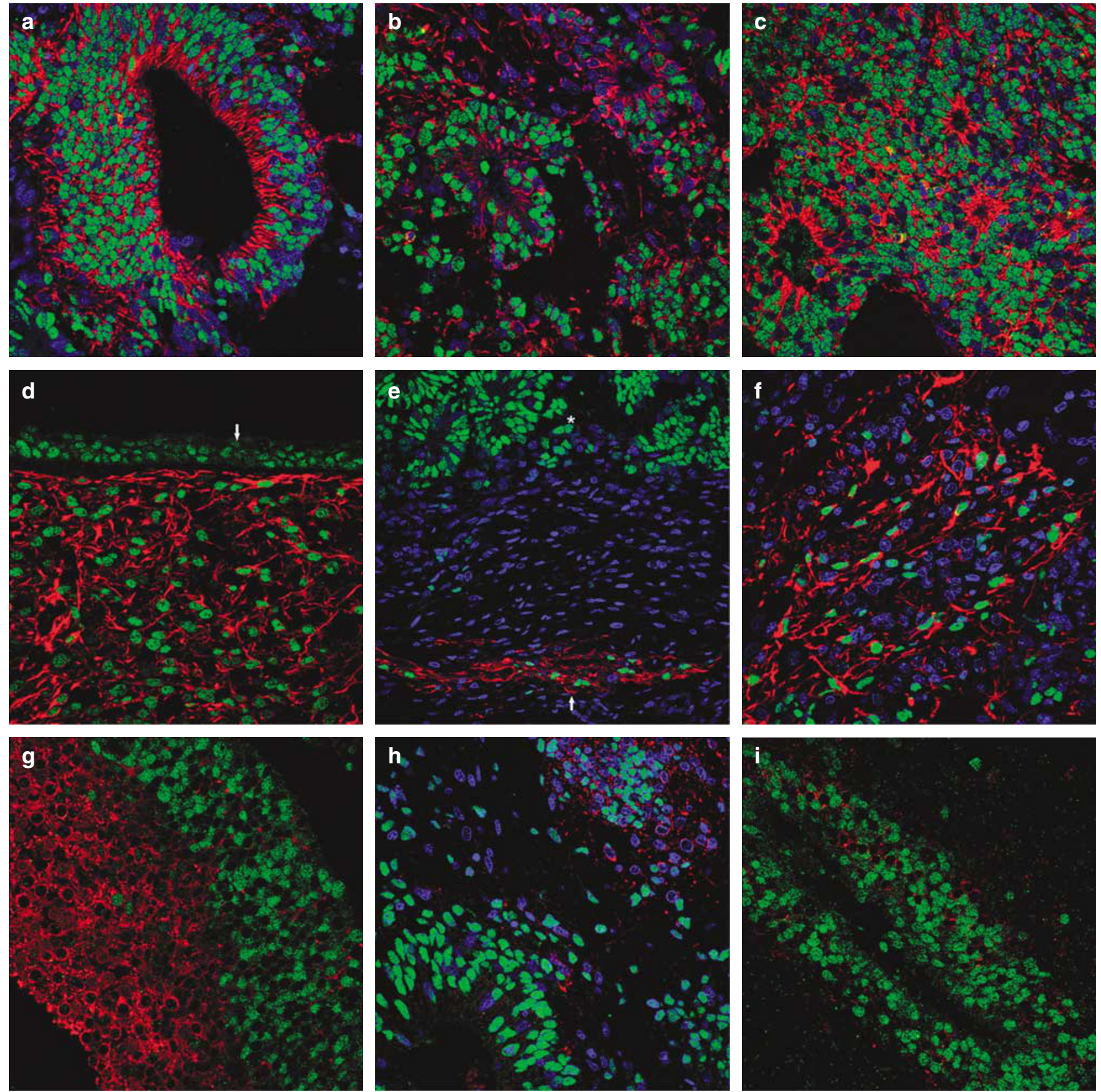

Figure 4 Immunofluorescence with double labeling for the phenotyping of Sox2-positive cells in central nervous system immature teratomas. (a) Sox2-positive cells in primitive neuroepithelial tubules coexpressed nestin, a neural stem cell marker (Sox2 green, nestin red, DAPI blue, $\times 400$ ); (b) Sox2-positive cells also expressed vimentin, another neural stem cell marker. Note the Sox2 ${ }^{-} /$vimentin $^{+}$cells outside neuroepithelial tubules (Sox2 green, vimentin red, DAPI blue, $\times 400$ ); (c) Sox2-positive cells in primitive neuroectodermal tumor-like areas also expressed nestin (Sox2 green, nestin red, DAPI blue, $\times 400$ ); (d) Sox2-positive cuboidal epithelium (arrow) did not express nestin, in contrast to neighboring Sox $2^{+} /$nestin $^{+}$neuroglial progenitors (Sox2 green, nestin red, $\left.\times 400\right) ;(\mathbf{e})$ and $(\mathbf{f})$ Sox2 $^{+} / \mathrm{GFAP}^{+}$ cells (arrow) were found in scattered areas remote from neuroepithelial tubule-rich areas (asterisk) (Sox2 green, GFAP red, DAPI blue; $\times 280$ and $\times 400$ ); (g) Tuj-1, an immature neuronal marker, was expressed in the periphery of neuroepithelial tubules. Tuj-1 and Sox2 expressions were found to be mutually exclusive (Sox2 green, Tuj-1 red, $\times 400$ ); (h) MAP2 (a mature neuronal marker) expression was observed mainly within cells surrounding primitive neuroepithelial tubules. MAP2 and Sox2 expressions were also mutually exclusive (Sox2 green, MAP2 red, DAPI blue, $\times 400$ ); (i) CNPase, a mature oligodendrocyte marker, was found within neuroepithelial tubules, and it and Sox2 expressions were also mutually exclusive (Sox2 green, CNPase red, $\times 400$ ).

(Figure 4d). GFAP-positive cell nests were observed at locations apart from neuroepithelial tubule-rich areas, which had an astroglial cell-like morphology with elongated processes, and many of these coexpressed Sox2 (Figure 4e and f).
Tuj-1, an immature neuronal marker, and MAP2, a mature neuronal marker, were expressed in the outer layer of neuroepithelial tubules, but the number of MAP2 positive cells was smaller. Sox2 expression and those of Tuj-1 and MAP2 were 
mutually exclusive (Figure $4 \mathrm{~g}$ and $\mathrm{h}$ ). Reactivity to CNPase, a mature oligodendrocyte marker was found in neuroepithelial tubules, and this too was not expressed with Sox2 (Figure 4i).

\section{Discussion}

Our study demonstrates that Sox2 is consistently expressed in the primitive neuroepithelial cells of central nervous system immature teratomas that comprise primitive neuroepithelial tubules and primitive neuroectodermal tumor-like areas. Mature tissue components were negative for Sox2, except for some cuboidal, columnar endodermal origin. Sox2 expression was found outside neuroepithelial tubules, which created a large Sox2-positive field. On the other hand, not all cells that composed neuroepithelial tubules expressed Sox2, which indicates that active cell fate commitment and neuroglial differentiation took place within and around primitive neuroepithelial tubules.

Immunofluorescence with double labeling revealed that the majority of Sox2-positive cells also expressed nestin and vimentin. Sox $2^{+} /$nestin $^{+} /$ vimentin $^{+}$cells were also abundant in primitive neuroectodermal tumor-like areas. These Sox2 ${ }^{+} /$ nestin ${ }^{+} /$vimentin $^{+}$cells represent neuroglial stem/ progenitor cell populations in the primitive neuroepithelial tissues of immature teratomas. The prognosis of immature teratomas of central nervous system depends on the presence and amounts of these tissues, which is reflected in the pathological grading of immature teratomas (so-called Norris grade). ${ }^{11-13}$ Recent evidences indicate that many tumors, including brain tumors, harbor a small population of cancer stem cells that eventually determine clinical course and prognosis. ${ }^{17-19}$ One important characteristic of cancer stem cells is that they resemble normal stem cells in the specific tissue in which the cancer originated. ${ }^{20}$ Sox $2^{+}$/ nestin ${ }^{+} /$vimentin $^{+}$cells in primitive neuroepithelial tissues can be considered as the cancer stem cells of immature teratomas, because they definitely constitute an aggressive cellular population in immature teratomas, and favor recurrence and a poor prognosis, and because they phenotypically resemble normal neural stem cells. This could partly explain the clinical relevance of Norris grade because it depends on the presence and amount of tissues enriched with presumptive cancer stem cells.

Some nestin ${ }^{+} /$vimentin $^{+}$cells within and around the primitive neuroepithelial tubules were Sox2 negative. This finding indicates that there may be a hierarchy of neural stem/progenitor cell markers, such as, of nestin and Sox2, and that Sox2-positive cells represent a more specific population of neuroglial progenitors.

Many GFAP-positive cells outside neuroepithelial tubules coexpressed Sox2, but Sox2-positive cells, wherever they were, did not the express neuronal markers, Tuj-1 and MAP2. Instead, a majority of Sox2-negative cells within and around primitive neuroepithelial tubules expressed these neuronal markers. This result supports the notion that active cell fate commitment and neuronal differentiation take place in and around primitive neuroepithelial tubules. A small portion of cells within neuroepithelial tubules expressed CNPase, an oligodendroglial marker, and CNPase and Sox2 expression were found to be mutually exclusive. These findings on cell fate commitment are consistent with those of previous studies in animal tissues, which revealed that Sox2 inhibits neurogenesis, but permits gliogenesis in neuroglial progenitor cells. Moreover, mature astrocytes retain Sox2 expression and even upregulate it when activated, but neurons do not express Sox $2 .^{7,9}$

One major difference observed between fetal brain and the primitive neuroepithelial tubules of immature teratomas was that many Sox $2^{+} / \mathrm{GFAP}^{+}$cells were found in the ventricular zone and subventricular zone of fetal brain, whereas virtually no Sox $2^{+}$/ $\mathrm{GFAP}^{+}$cells were observed in the primitive neuroepithelial tubules of immature teratomas. Only mature astroglia-like cells located apart from primitive neuroepithelial tissues displayed Sox $2^{+} / \mathrm{GFAP}^{+}$ phenotype in immature teratomas. This fact may simply reflect the disordered, pathological development of primitive neuroepithelial tissues towards a neuronal fate in immature teratomas. However, it is also possible that differences in GFAP expression are related to the variable developmental stages of tumor and normal fetal brain neuroepithelial tissues, because the oligodendroglial marker, CNPase, was expressed in the neuroepithelial tubules of immature teratomas.

In addition to neuroepithelial cells, epithelium of endodermal origin was also found to express Sox2. Some reports indicate that Sox2 is involved in endodermal lineage differentiation. In one study, Sox2 was found to be expressed in early chick endodermal epithelium from pharynx to lung and stomach. $^{21}$ Other studies on human specimens showed that Sox2 is expressed in normal stomach epithelium, ${ }^{22}$ and in cancers of endodermal origin tissues. ${ }^{23}$ However, little is known about the role of Sox2 during endodermal differentiation.

The diagnosis of immaturity is critically important for the management of patients with teratomas of central nervous system, because the recurrence of immature teratomas is not uncommon, and thus, they require closer follow-up and adjuvant treatment after surgical resection. ${ }^{13,15,16}$ Since teratomas have a highly heterogeneous composition and immature neuroepithelial tissue is not uniformly distributed, but usually confined to some part of the whole tumor, a risk of underdiagnosis exists. Furthermore, as described above, immature teratoma pathological grading is essentially quantitative, and is based on an estimate of primitive neuro- 
epithelial tissues. Thus, if a reliable immunohistochemical marker for neuroepithelial tissues is available, the accuracy of immature teratoma diagnosis and quantitative grading can be improved. In the present study, Sox2 was found to be a sensitive marker of immature neuroepithelial tissue, but not a specific marker, because differentiated astroglial cells and some endodermal epithelium were also observed to express Sox2. Nonetheless, even primitive neuroepithelial cells, which do not constitute apparent neuroepithelial tubules, were more readily discernible by Sox2 immunohistochemistry than by conventional hematoxylin and eosin staining. Therefore, Sox2 could help to prevent underdiagnoses and improve the quantitative grading of central nervous system immature teratomas, provided the above-mentioned exception concerning differentiated astroglial cells and endodermal epithelium is taken into account.

In conclusion, Sox2 was found to be highly expressed in the primitive neuroepithelial tissues of immature teratomas of central nervous system, whereas its expression in other mature tissue was limited to some epithelium of endodermal origin. High Sox2 expression in the primitive neuroepithelial tissue, which is the most aggressive component within immature teratomas, makes this transcription factor a good marker for proper diagnosis and quantitative pathological grading.

\section{Acknowledgement}

This study was supported by a grant (no. 7-20051216) from the Korean Ministry of Health and Welfare.

\section{Disclosure/conflict of interest}

None.

\section{References}

1 Rao MS, Jacobson M. Developmental neurobiology, 4th edn. Kluwer Academic/Plenum Publishers: New York, 2005.

2 Takahashi T, Goto T, Miyama S, et al. Sequence of neuron origin and neocortical laminar fate: relation to cell cycle of origin in the developing murine cerebral wall. J Neurosci 1999;19:10357-10371.

3 Bertrand N, Castro DS, Guillemot F. Proneural genes and the specification of neural cell types. Nat Rev Neurosci 2002;3:517-530.

4 Gubbay J, Collignon J, Koopman P, et al. A gene mapping to the sex-determining region of the mouse $\mathrm{Y}$ chromosome is a member of a novel family of embryonically expressed genes. Nature 1990;346: $245-250$.
5 Boyer LA, Lee TI, Cole MF, et al. Core transcriptional regulatory circuitry in human embryonic stem cells. Cell 2005;122:947-956.

6 Bylund M, Andersson E, Novitch BG, et al. Vertebrate neurogenesis is counteracted by Sox1-3 activity. Nat Neurosci 2003;6:1162-1168.

7 Graham V, Khudyakov J, Ellis P, et al. SOX2 functions to maintain neural progenitor identity. Neuron 2003; 39:749-765.

8 Catena R, Tiveron C, Ronchi A, et al. Conserved POU binding DNA sites in the Sox2 upstream enhancer regulate gene expression in embryonic and neural stem cells. J Biol Chem 2004;279:41846-41857.

9 Bani-Yaghoub M, Tremblay RG, Lei JX, et al. Role of Sox2 in the development of the mouse neocortex. Dev Biol 2006;295:52-66.

10 Ellis P, Fagan BM, Magness ST, et al. SOX2, a persistent marker for multipotential neural stem cells derived from embryonic stem cells, the embryo or the adult. Dev Neurosc 2004;26:148-165.

11 Kooijman CD. Immature teratomas in children. Histopathology 1988;12:491-502.

12 Norris HJ, Zirkin HJ, Benson WL. Immature (malignant) teratoma of the ovary: a clinical and pathologic study of 58 cases. Cancer 1976;37:2359-2372.

13 Phi JH, Kim SK, Park SH, et al. Immature teratomas of the central nervous system: is adjuvant therapy mandatory? J Neurosurg 2005;103:524-530.

14 Chambers I, Smith A. Self-renewal of teratocarcinoma and embryonic stem cells. Oncogene 2004;23:71507160.

15 Sawamura Y, Kato T, Ikeda J, et al. Teratomas of the central nervous system: treatment considerations based on 34 cases. J Neurosurg 1998;89:728-737.

16 Matsutani M, Sano K, Takakura K, et al. Primary intracranial germ cell tumors: a clinical analysis of 153 histologically verified cases. J Neurosurg 1997;86: 446-455.

17 Bonnet D, Dick JE. Human acute myeloid leukemia is organized as a hierarchy that originates from a primitive hematopoietic cell. Nat Med 1997;3: 730-737.

18 Singh SK, Clarke ID, Terasaki M, et al. Identification of a cancer stem cell in human brain tumors. Cancer Res 2003;63:5821-5828.

19 Galli R, Binda E, Orfanelli U, et al. Isolation and characterization of tumorigenic, stem-like neural precursors from human glioblastoma. Cancer Res 2004;64:7011-7021.

20 Vescovi AL, Galli R, Reynolds BA. Brain tumour stem cells. Nat Rev Cancer 2006;6:425-436.

21 Ishii Y, Rex M, Scotting PJ, et al. Region-specific expression of chicken Sox2 in the developing gut and lung epithelium: regulation by epithelial-mesenchymal interactions. Dev Dyn 1998;213:464-475.

$22 \mathrm{Li}$ XL, Eishi Y, Bai YQ, et al. Expression of the SRYrelated HMG box protein SOX2 in human gastric carcinoma. Int J Oncol 2004;24:257-263.

23 Sanada Y, Yoshida K, Ohara M, et al. Histopathologic evaluation of stepwise progression of pancreatic carcinoma with immunohistochemical analysis of gastric epithelial transcription factor SOX2: comparison of expression patterns between invasive components and cancerous or nonneoplastic intraductal components. Pancreas 2006;32:164-170. 